Objectives: To assess early signs of CKD risk development in patients with gout by SENS-CRS technology.

Methods: We examined 66 patinets with gout. 95\% were men (average age $54 \pm 9$ years). Duration of gout was 8 [4; 11] years. All patients had chronic gouty arthritis, $30 \%$ of patients had tophuses. SENS-CRS was carried out on a 2-detector $\gamma$-camera with simultaneous recording in 2 projections. In studies of the urinary system domestic radiopharmaceutical (RP) 99mTc-technefor with glomerulo- and $10-15 \%$ tubulotropic properties was used. The CRS study consisted of: 1) a basic 21-min functional study; 2) a delayed 21-min study (without the injection of RP) with a preliminary diuresis forcing to identify persistent urodynamic dysfunction.

Results: 16 patients with gout with serum creatinine level of more than $125 \mu \mathrm{mol} / / \mathrm{had}$ established diagnosis of chronic pyelonephritis, glomerulonephritis, urolithiasis, etc. and were allocated into a separate subgroup. In the comparative analysis of these 16 patients and other 50 patients with gout there was reliable distinction only for one indicator D (\%), the rate of RP removal from a kidney parenchyma $(p<0,05)$. There was no significant distinctions for glomerular filtration rate by Reberg, the nuclear indicator $\left(\mathrm{G}_{\mathrm{ren}}\right)$ of renal parenchyma concentration function, and other indicators. Renocortical parameter D (\%) is one of the earliest highly sensitive markers of intrarenal developments of stagnation at development of serious morphofunctional violations in a parenchyma. In the subgroup of 16 patients the indicator accounted on average $D=48 \% \pm 7 \%$ (range $38-55 \%)$; in subgroup of 50 patients $D=63 \% \pm 10 \%(47-89 \%)$.

According to the CKD-EPI formula and SENS-CRS technology in the subgroup of 16 patients CKD stage II-III was evaluated. In addition to the sign of broken rate of RP removal from a parenchyma (D) when comparing the 2 phases of CRS there was observed a steady sign of stagnation in a parenchyma and groups of upper and/or lower cups: ID = $\mathrm{G}_{\mathrm{calc}} / \mathrm{GC}<1$. Based on the water test which accelerates diuresis there was a sufficient regulation of urostasis in the renal pelvis revealed at the basic test (IP $\left.=G_{\text {pelv }} / G P>1,0\right)$. This indicated that the functional reserve of the outflow regulation from the kidneys was preserved at these patients.

Conclusion: This functional diagnostics allows one-time control of hemodynamics and concentration function of the parenchyma of each kidney, quantitative and qualitative signs of urodynamic delays in the intra- and post-renal urinary tract. Differentiated analysis SENS-CRS contributes to the timely therapeutic correction as well as referral for consultation to specialists (urologists, nephrologists, gynecologists, etc.).

Disclosure of Interests: Margarita Gromova Speakers bureau: Speaker during conferences in Russian Federation, Vladimir Tsurko Speakers bureau: Speaker during conferences in Russian Federation, Anna Kashkadayeva: None declared, Svetlana Averinova: None declared, Andrey Aliokhin: None declared

DOI: 10.1136/annrheumdis-2019-eular.755

\section{AB0872 OXIDATIVE STRESS MARKER ALLANTOIN IS NOT ASSOCIATED WITH THE CHANGE OF SERUM URIC ACID LEVEL IN PATIENTS WITH SYSTEMIC RHEUMATIC DISEASES AFTER ABROGATION OF SYSTEMIC INFLAMMATION BY TNF INHIBITION}

Lenka Hasikova $^{1,2}$, Marketa Pavlikova $^{3}$, Petr Kozlik ${ }^{4}$, Kveta Kalikova $^{5}$,

Blanka Stiburkova ${ }^{1,6}$, Jakub Zavada ${ }^{1,2}{ }^{1}$ Institute of Rheumatology, Prague, Czech Republic; ${ }^{2}$ Department of Rheumatology, First Faculty of Medicine, Charles University, Prague, Czech Republic; ${ }^{3}$ Department of Probability and Mathematical Statistics, Faculty of Mathematics and Physics, Charles University, Prague, Czech Republic; ${ }^{4}$ Department of Analytical Chemistry, Faculty of Science, Charles University, Prague, Czech Republic; ${ }^{5}$ Department of Physical and Macromolecular Chemistry, Faculty of Science, Charles University, Prague, Czech Republic; ${ }^{6}$ Department of Pediatrics and Adolescent Medicine, First Faculty of Medicine, Charles University and General University Hospital in Prague, Prague, Czech Republic

Background: In patients with gout, the serum uric acid (SUA) is usually lower during acute gouty attacks than during intercritical periods. In a previous study, we have shown that abrogation of systemic inflammation by TNF inhibitors (TNFi) results in an increase in the levels of SUA in patients with systemic rheumatic diseases. We have not found any correlation between the magnitude of change of SUA and CRP or pro-inflammatory cytokines (MCP-1, IFN- $\alpha 2$, IFN- $\gamma$, II-1 $\beta$, IL-6, IL-8, IL-10, IL-12, IL17a, IL-18, IL-23, IL-33, TNF- $\alpha) .{ }^{1}$ Another possible mechanism for the lowering of SUA during inflammation may be consumption of circulating SUA in free radical reactions generated during systemic inflammation. Allantoin has been validated as a stable biomarker of oxidative stress in humans. $^{2}$
Objectives: We aimed to investigate whether the magnitude of change of SUA after starting therapy with TNFi is associated with the change of oxidative stress marker - allantoin in patients with systemic autoimmune rheumatic diseases: rheumatoid arthritis (RA), ankylosing spondylitis (AS), psoriatic arthritis (PSA) and juvenile idiopathic arthritis (JIA)

Methods: A cohort of 94 patients with clinically active chronic inflammatory rheumatic diseases (31 with RA, 33 with AS, 18 with PsA, 12 with $\mathrm{JIA}$ ) and CRP $>10 \mathrm{mg} / \mathrm{L}$ was recruited in the Institute of Rheumatology, Prague. SUA, CRP and allantoin were measured before and after 3 months of treatment with TNFi. Assessment of allantoin in plasma samples was carried out on the Agilent Infinity 1290 system coupled with a Triple Quad 6460 tandem mass spectrometer. For the statistical analysis the ratio between the values at the baseline and values after 3 months of therapy with TNFi were used. We retrieved demographic data and disease characteristics.

Results: The levels of SUA have significantly increased after 3 months of treatment with TNFi (270.5 [78.8] vs. 303.0 [101.5] $\mu \mathrm{mol} / \mathrm{l}, \mathrm{p}<$ 0.0001), while CRP (29.4 [27.7] vs. 2.0 [3.8] mg/l, $p<0.0001)$ has decreased. There was no significant change of allantoin before and after 3 months of treatment with TNFi. In the linear regression model, CRP or allantoin had no effect on the magnitude of change of SUA.

Conclusion: The abrogation of systemic inflammation by TNFi results in an increase in the levels of SUA in patients with systemic rheumatic diseases, but the mechanism remains elusive. We have not observed any correlation between the magnitude of change of SUA and CRP or oxidative stress marker allantoin

\section{REFERENCES}

[1] Petru L, Hulejova H, Mahajan A, et al. SAT0366 Serum uric acid increases after abrogation of systemic inflammation by TNF inhibition. Annals of the Rheumatic Diseases 2018;77:1047

[2] Marrocco I, Altieri F, Peluso I. Measurement and Clinical Significance of Biomarkers of Oxidative Stress in Humans. Oxidative medicine and cellular longevity 2017;2017:6501046

Acknowledgement: This work was supported by the Charles University research grant GA UK No.940517, by the Ministry of Health, Czech Republic (Institute of Rheumatology - project for conceptual development of a research organisation, 00023728) and by the Czech Science Foundation, Grant No 19-18005Y.

Disclosure of Interests: Lenka Hasikova: None declared, Marketa Pavlikova: None declared, Petr Kozlik: None declared, Kveta Kalikova: None declared, Blanka Stiburkova: None declared, Jakub Zavada Consultant for: Genzyme-Sanofi, Glaxo, Pfizer, Abbvie, Speakers bureau: Novartis, Sandoz, Biogen

DOI: 10.1136/annrheumdis-2019-eular.7112

\section{AB0873 ENDOTHELIAL DYSFUNCTIONIN PATIENTS WITH GOUT. RELATIONSHIP BETWEEN CARDIOVASCULAR RISK FACTORS}

Ekaterina llinykh, Maxim Eliseev, Alexander Volkov. V.A.Nasonova Research Institute of Rheumatology, Moscow, Russian Federation

Background: Patients with gout have a high risk of developing cardiovascular disease based on atherosclerosis. An early marker, and at the same time a risk factor of cardiovascular disease, is a violation of the vasoregulating endothelial activity.

Objectives: To identify the relationship between cardiovascular risk factors and endothelial dysfunction (ED) in patients with gout.

Methods: The study included 80 pts with gout. The criteria for inclusion were 1.Male

2.Age $>55$ years 3 . Interictal period of arthritis 4 .Absence of clinical signs of coronary artery disease 5.Absence of drug therapy. Vasoregulating endothelial activity was evaluated in all pts, 52 of them determined the carotid intima-media thickness (C-IMT). Flow-mediated (endotheliumdependent) dilatations (FMD), nitroglycerin-induced (endothelium - independent) dilatation (EID) were assessed by highresolution ultrasonography (D. Celermajer). A non-invasive ultrasound technique was used to measure C-IMT. All patients were diagnosed with serum total cholesterol (CHOL), subtypes (LDL-C, HDL-C), glucose (GLU), uric acid (SUA), hsCRP. Cardiovascular risk (CVR) was calculated. Statistical analysis was conducted using the applied programs package of descriptive statistics STATISTICA 12.0 (StatSoft.Inc.,USA).

Results: It was found that $41(51.25 \%)$ pts had FMD less than $8 \%$. A correlation was found between FMD and CVR $(r=-0.28, p<0.05)$, age $(r=-0.37, p<0.001)$, Body mass index (BMI) 
$(r=-0.23, p<0.05)$. The links between FMD and LDL, GLU, SUA and with parameters reflecting the severity of gout were not revealed $(p>0.05)$. C-IMT in pts was $0.7(0.6-0.8) \mathrm{mm} .7(14,4 \%)$ pts had $>1.0$ $\mathrm{mm}$. A direct correlation was found between the C-IMT and the FMD ( $p$ $<0.01)$, CVR $(p<0.001)$, age $(p<0.001)$, duration of gout $(p<0.001)$, the number of joints affected for all time $(p<0.005)$ and the frequency of arthritis in the last year $(p<0.05)$. Correlation between C-IMT and LDL, GLU, SUA, hsCRB was not revealed ( $p>0.05$ ).

Conclusion: The ED, reflecting the risk of atherosclerosis, was found in $51.3 \%$ of patients with gout under the age of 55 years without clinical signs of coronary artery disease. The ED is associated with an increase in the CVR. C-IMT correlates with an increase in CVR and with parameters reflecting the severity of gout.

\section{REFERENCES}

[1] Celermajer D.S, Sorensen K.E., Gooch V.M. et al. Non-invasive detection of endothelial dysfunction in children and adults at risk atherosclerosis// Lancet.- 1992. - Vol.340. - P.1111-1115.

[2] Corretti M.C., Anderson T.J., Benjamin E.J. et al. Guidelines for the ultrasound assessment of endothelial-dependent flow-mediated vasodilation of brachial artery//Jam. Coll. Cardiol. - 2002. - Vol.39. - P.257-265.

Disclosure of Interests: None declared

DOI: 10.1136/annrheumdis-2019-eular.6744

\section{AB0874 ANALYSIS OF LIFESTYLE AND CLINICAL FEATURES OF THE PATIENTS WITH GOUT IN MEIZHOU, GUANGDONG, CHINA}

Yutong Jiang ${ }^{1}$, Yiquan Wen ${ }^{2}$, Zhongyu Liu' ${ }^{1}$, Qiyun Chen ${ }^{2}$, Yefei Huang ${ }^{1}$, Yunfeng Pan ${ }^{1}$, Jieruo Gu${ }^{1} .{ }^{1}$ the Third Affiliated Hospital of Sun Yat-sen University, Guangzhou, China; ${ }^{2}$ The Third Affiliated Hospital of Sun Yat-sen University, Yuedong Hospital, Meizhou, China

Background: Gout is one of the most common metabolic diseases caused by purine metabolic disorder, leading to joint destruction and kidney impairment. With the improvement of living conditions, the incidence of gout is increasing year by year, especially in the developed coastal areas of China. Previous studies found that the management of gout was unsatisfied [1]. Besides, the development of rheumatology in Meizhou, Guangdong province is extremely slow. There are only ten rheumatologists, and the general public has a limited understanding of gout.

Objectives: Our aim was to explore the lifestyle, clinical features and risk factors of recurrent gout attacks in patients with gout in Meizhou, Guangdong province.

Methods: Demographic data, lifestyle and clinical data of 188 patients with gout in Meizhou were collected. Demographic variables included age, gender, marital status, education, BMI, smoking status, drinking status, diet, daily water intake, work style, exercise habit, late sleeping habit. Smoking status includes never smoking, ever-smoking or current smoking and pack-year of smoking. The diet includes loving sweet food, beverage, seafood, fried food, and disliking vegetables and fruits. Amount of daily water intake is divided into $500-999 \mathrm{ml}, 1000-1499 \mathrm{ml}, 1500-1999 \mathrm{ml}$ and $2000 \mathrm{ml}$ and above. Working styles include long time of sitting, standing, walking, etc. Exercise habits include not exercising, exercising at least 1 hour a day, exercising at least 1 hour a week, and exercising at least 1 hour a month. Clinical characteristics include first time and cause of gout attack, blood uric acid value, the position of gout attack, pain score duration, alleviating methods, treatment, patients' self-reported gout episodes (below 5 times, 5-10 times, 11-20 times, more than 20 times), tophi, family history and comordities. Multiple regression analysis was used to analyze the factors of the numbers of gout attacks.

Results: of the 188 cases, $171(91.0 \%)$ were males and $17(9.0 \%)$ were females. The average age was $55 \pm 16$ years. $94(50 \%)$ of the patients were drinking alcohol, and $58(30.9 \%)$ were fond of seafood and other high-purine food. 149 (79.3\%) patients had less than $1000 \mathrm{ml}$ of water per day, and the vast majority of patients had no exercise habit (136 $(72.3 \%))$. The interval between the first gout attack and the first uric acid increasing was $-0.4(\mathrm{~S}=2.5)$ years. The first gout attack occurred in the first metatarsophalangeal joint in $57(30.3 \%)$ cases, followed by ankle jointa in $40(21.3 \%)$ cases, knee jointa in $23(12.2 \%)$ cases, and dorsa foot joints in $23(12.2 \%)$ cases. The first gout attack occurred in 87 $(46.3 \%)$ cases on the right side of the body. 25 (13.3\%) patients' firs gout attack occurs in more than one joint. The number of tophus is 0 21 per person. $64(38.1 \%)$ patients had more than 20 gout attacks. Up to $70(40.7 \%)$ patients never visited the hospital. 14 people were hospitalized due to gout. Colchicine was the most commonly used drug in treatment in $59(31.4 \%)$ cases, followed by allopurinol in $49(26.1 \%)$ cases. Gout was positively correlated with age $(B=0.313, P=0.007)$ and uric acid $(B=0.04, P=0.018)$.

Conclusion: Gout patients in Meizhou, Guangdong are mainly male, and have bad living habits such as drinking, loving hyper purine food and lack of drinking water and doing exercises. The first gout attack is more likely to involve a unilateral lower extremity, especially first metatarsophalangeal joints. Meizhou patients with gout have earlier occurrence of tophi, and fail to see a doctor and have medication regularly. Therefore, gout patients should develop good living habits and receive regular medical education.

\section{REFERENCE}

[1] Li XM, Chen ZN, Gu JR. The current situation of hyperuricemia cannot be ignored [J]. New medicine,2016(03): 137-141 (in Chinese).

Disclosure of Interests: None declared

DOI: 10.1136/annrheumdis-2019-eular.609

\section{AB0875 GOUT, NOT JUST A DISEASE OF THE FOOT. LITERATURE REVIEW OF SYSTEMIC DEPOSITION OF} URATE

Ada Kumar ${ }^{1}$, Puja Khanna ${ }^{2} .{ }^{1}$ Horizon Pharma, Lake Forest, United States of America; ${ }^{2}$ University of Michigan, Ann Arbor, United States of America

Background: Gout is the most common adult inflammatory arthropathy in the US. Although tophi in the extremities is a known source of the inflammatory cascade, urate deposition in organs throughout the body is not as well recognized. Patients with gout often have associated co-morbidities including renal disease, cardiovascular disease and metabolic syndrome, however, a casual role has not been established. Direct urate deposition in these organ systems may be of interest to link the causality of these systemic disorders.

Objectives: Perform a literature review including clinical exam, autopsy, pathology, and radiology imaging results demonstrating systemic deposition of urate exclusive of the extremities.

Methods: PUBMED from 1920 to 2018 was searched to identify reports of non-extremity urate deposition. Key words included: extra-articular gout, systemic deposition of urate, ocular gout, gout nephropathy, renal tophi, gouty heart, cardiac valves and urate, urate deposition in the arteries, prostate and urate, autopsy findings in gout, cutaneous urate deposits, gouty panniculitis, auricular gout, breast and urate, gastrointestinal gout, pancreas and tophus, laryngeal tophus, and spinal gout. The reference lists from these publications were also used to identify additional articles. The literature was reviewed for organ system involvement and documented based on sites of urate deposition within an organ system.

Results: There were 249 case reports documenting non-extremity urate deposition confirmed by autopsy, biopsy, surgery, clinical exam and/or radiology imaging. Urate deposition was reported in multiple organ sys tems (Table 1) including the spine, integumentary, ocular, renal, cardiovascular, gastrointestinal, larynx, breast, middle ear, pancreas, nasal, prostate gland, liver, pulmonary, penis, nailbed, and pelvis.

Conclusion: Numerous case reports document systemic deposition of urate based on autopsy, pathology, imaging and clinical exam. Urate crystal deposition with the formation of tophi and micro-tophi involve multiple organ systems including cardiovascular, renal, spine, integumentary, prostate, bowel, pancreas, eyes, pelvic, breast, lungs, middle ear, larynx, liver, penis, nailbed, and nose. Given the strong association of gout with various comorbidities, this demonstrates a need for further studies to determine the clinical significance of systemic urate deposition with respect to ongoing subclinical inflammation and potential end-organ damage.

\section{REFERENCES}

[1] Park, J. et al. BMJ Open. 2014; 4:e005308.

[2] Nickeleit, V, et al. Nephrol Dial Transplant. 1997; 12:1832-1838.

[3] Ao, J, et al. Clinical and Experimental Ophthalmology. 2017; 45: 73-80

[4] Forbess LJ, et al. Semin Arthritis Rheum. 2012; 42:146-54.

[5] Elgafy, H, et al. World J Orthop. 2016; 7(11): 766-775.

Abstract AB0875 Table 1

\begin{tabular}{ccc}
\hline Organ System & \# of Case & Diagnostics \\
Reports & (Autopsy, biopsy, clinical \\
$\mathrm{N}=249$ & exam, surgery, imaging) \\
\hline
\end{tabular}

